\section{Redaktionen har modtaget:}

DAVID ABULAFIA: A Mediterranean Emporium: The Catalan Kingdom of Majorca. Cambridge, New York \& Melbourne: Cambridge University Press 1994. 292 sider. ISBN 0-521-32244-8 hardback, pris £35.00.

EDUARDO P. ARCHETTI (ed.): Exploring the Written: Anthropology and the Multiplicity of Writing. Oslo Studies in Anthropology. Oslo: Scandinavian University Press 1994. 342 sider. ISBN 82-00-03937-4 hardback, pris NOK 298.

ARGONOTER - Nyhedsblad for Antropologforeningen i Danmark. Nr. 5+6, 1994. 20 sider, illustreret. Løssalg $15 \mathrm{kr}$. Udgiver: Antropologforeningen i Danmark (AID), Institut for Antropologi, Frederiksholms Kanal 4, 1220 K. Tlf: 35323472.

CLAUDIO BOGANTES (ed.): Memorias del VI Congreso del Grupo de Trabajo de Estudios Regionales del CEISAL. Århus: Center for Latinamerikastudier, Aarhus Universitet 1994. 244 sider.

VIGDIS BROCH-DUE, INGRID RUDIE \& TONE BLEIE (eds.): Carved Flesh/Cast Selves: Gendered Symbols and Social Practices. Cross-Cultural Perspectives on Women, vol. 8. Oxford \& Providence, R.I.: Berg Publishers 1994. 297 sider. ISBN 0-85496$725-7$ hardback, pris $£ 40.00$. ISBN 0-85496868-7 paperback, pris $£ 12.95$.

ELIZABETH M. BRUMFIELD \& JOHN W. FOX (eds.): Factional Competition and Political Development in the New World. New Directions in Archaeology. Cambridge, New York \& Victoria: Cambridge University Press 1994. 208 sider, illustreret. ISBN 0521-38400-1 hardback, pris $£ 32.50$.

ERNEST S. BURCH, Jr. \& LINDA J. ELLANNA (eds.): Key Issues in HunterGatherer Research. Explorations in Anthropology. Oxford \& Providence, R.I.: Berg Publishers 1994. 534 sider. ISBN 0-85496$375-8$ hardback, pris $£ 59.95$. ISBN 0-85496376-6 paperback, pris $£ 19.95$.
PAULINE BURTON, KETAKI KUSHARI DYSON \& SHIRLEY ARDENER (eds.): Bilingual Women: Anthropological Approaches to Second-Language Use. Cross-Cultural Perspectives on Women, vol. 9. Oxford \& Providence, R.I.: Berg Publishers 1994. 210 sider. ISBN 0-85496-737-0 hardback, pris $£ 34$.95. ISBN 0-85496-864-4 paperback, pris $£ 14.95$.

N. G. BUTLIN: Economics and the Dreamtime: A Hypothetical History. Cambridge, New York \& Victoria: Cambridge University Press 1994. 384 sider, illustreret. ISBN 0521-43236-7 hardback, pris £45.00. ISBN 0521-43820-9 paperback, pris $£ 15.95$.

COMPANION ENCYCLOPEDIA OF ANTHROPOLOGY: Humanity, Culture and Social Life. Tim Ingold (ed.). London \& New York: Routledge 1994. 1127 sider. ISBN 0415-02137-5 hardback, pris £85.00.

JEREMY COOTE \& ANTHONY SHELTON (eds.): Anthropology, Art, and Aesthetics. Oxford Studies in Social and Cultural Anthropology. Oxford: Oxford University Press 1994. 296 sider, illustreret. ISBN 0-19827945-0 paperback, pris $£ 12.95$.

DEN NY VERDEN nr. 1, 1994. Tema: Indfødte folk $i$ Afrika. Temaredaktør: Hanne Veber. 128 sider. Pris 75 kr. Årsabonnement 1994 (4 nr.) 195 kr. Udgiver: Center for Udviklingsforskning, Gl. Kongevej 5, $1610 \mathrm{~K} \varnothing-$ benhavn $\mathrm{V}$.

PHILIPPE DESCOLA: In the Society of $\mathrm{Na}$ ture: A Native Ecology in Amazonia. Cambridge Studies in Social and Cultural Anthropology no. 93. Cambridge, New York \& Melbourne: Cambridge University Press 1993. 372 sider, illustreret. ISBN 0-52141103-3 hardback, pris $£ 45.00$.

CHRISTOPHER DUGGAN: A Concise History of Italy. Cambridge Concise Histories Series. Cambridge, New York \& Melbourne: Cambridge University Press 1994. 320 sider, illustreret. ISBN 0-521-40285-9 hardback, pris £25.00. ISBN 0-521-40848-2 paperback, pris $£ 8.95$. 
DAISY DWYER \& JUDITH BRUCE (eds.): A Home Divided - Women and Income in the Third World. Stanford: Stanford University Press 1994 (1988). Distributed by Cambridge University Press. 289 sider. ISBN 08047-2213-7 paperback, pris $£ 10.95$.

MICHAEL D. FISCHER: Applications in Computing for Social Anthropologists. ASA Research Methods. London \& New York: Routledge 1994. 233 sider, illustreret. ISBN 0-415-01818-8 hardback, pris $£ 37.50$, ISBN 0-415-01819-6 paperback, pris $£ 13.99$.

JOHN GLEDHILL: Power and its Disguises: Anthropological Perspectives on Politics. London \& Boulder: Pluto Press 1994. 248 sider. ISBN 0-7453073-96 paperback, pris £9.95. ISBN 0-7453073-88 hardback, pris $£ 35.00$.

MICHAEL HARBSMEIER: Wilde Völkerkunde: Andere Welten in deutschen Reiseberichten der Frühen Neuzeit. Frankfurt/ New York: Campus Verlag 1994. Historische Studien Band 12. 330 sider. ISBN 3-59335095-5, pris DM 68.

SIDNEY L. HARRING: Crow Dog's Case: American Indian Sovereignty, Tribal Law, and United States Law in the Nineteenth Century. Cambridge Studies in the North American Indian History. Cambridge, New York \& Melbourne: Cambridge University Press 1994. 300 sider, illustreret. ISBN 0521-41563-2 hardback, pris $£ 35.00$. ISBN 0521-46715-2 paperback, pris $£ 17.95$.

ULLA HASAGER \& JONATHAN FRIEDMAN (eds.): Hawai'i - Return to Nationhood. IWGIA Document no. 75. Copenhagen: International Work Group for Indigenous Affairs 1994. 328 sider, illustreret. Pris US\$33.00.

JORDENS FOLK nr. 1, 1994. Tema: FNs Familieår. Nr. 2 (uden tema). 40 sider, illustreret. Løssalg $45 \mathrm{kr}$. Årsabonnement $160 \mathrm{kr}$. Henvendelse: Frank Sejersen, Institut for Antropologi, Frederiksholms Kanal 4, $1220 \mathrm{~K}$. Tlf: 35323472 .
IAN KEEN: Knowledge and Secrecy in an Aboriginal Religion: Yolngu of North-East Arnhem Land. Oxford Studies in Social and Cultural Anthropology. Oxford: Clarendon Press. 368 sider, illustreret. ISBN 0-19827900-0 hardback, pris $£ 35.00$.

PETER LASSMAN \& RONALD SPEIRS (eds.): Weber: Political Writings. Cambridge Texts in the History of Political Thought. Cambridge, New York \& Melbourne: Cambridge University Press 1994. 390 sider. ISBN 0-521-39312-4 hardback, pris £27.95. ISBN 0-521-39719-7 paperback, pris £14.95.

JOHN LIEP \& KAREN FOG OLWIG (red.): Komplekse liv: Kulturel mangfoldighed $i$ Danmark. København: Akademisk Forlag 1994. 182 sider. Pris 198 kr.

SARAH LUND SKAR: Lives Together Worlds Apart: Quechua Colonization in Jungle and City. Oslo Studies in Anthropology. Oslo: Scandinavian University Press 1994. 290 sider, illustreret. ISBN 82-0021957-7 hardback, pris NOK $298 \mathrm{kr}$.

MARYON McDONALD (ed.): Gender, Drink and Drugs. Cross-Cultural Perspectives on Women, vol. 10. Oxford \& Providence, R. I.: Berg Publishers 1994. 270 sider. ISBN 0-85496-719-2 hardback, pris £34.95. ISBN 0-85496-867-9 paperback, pris $£ 14.95$.

ELINOR G. K. MELVILLE: A Plague of Sheep. Studies in Environment and History. Cambridge, New York \& Melbourne: Cambridge University Press 1994. 203 sider. ISBN 0-521-42061-X hardback, pris £35.00.

DANIEL MILLER: Modernity - An Ethnographic Approach. Dualism and Mass Consumption in Trinidad. Explorations in Anthropology Series. Oxford \& Providence, R.I.: Berg Publishers 1994. 288 sider. ISBN 0-85496-916-0 hardback, pris $£ 39.95$. ISBN 0-85496-917-9 paperback, pris $£ 14.95$. ISBN 0-521-46637-7 paperback, pris £9.95.

INGRID RUDIE; Visible Women in East Coast Malay Society: On the Reproduction of 
Gender in Ceremonial, School and Market. Oslo Studies in Anthropology. Oslo: Scandinavian University Press 1994. 337 sider. ISBN 82-00-21919-4 hardback, pris NOK 298.

REGINA SCHULTE: The Village in Court: Arson, Infanticide, and Poaching in the Court Records of Upper Bavaria, 18481910. Cambridge, New York \& Melourne: Cambridge University Press 1994. 176 sider. ISBN 0-521-43186-7 hardback, pris $£ 40.00$.

SFINX, 17. årgang, nr. 1 (uden tema) \& nr. 2 (tema: Cypern), 1994. 96 sider, rigt illustreret. Løssalg 50 kr.. Årsabonnement (4 nr.) 152 kr. Henvendelse: SFINX, Klassisk Arkæologi, Aarhus Universitet, 8000 Århus C. Tlf: 86192607 .

Karlighed og (post)moderne tider. Temanummer af TENDENS - Tidsskrift for Kultursociologi. 6. årgang, nr. 1, 1994. 106 sider, illustreret. Løssalg 75 kr. Abonnement (2 nr.) $150 \mathrm{kr}$. Henvendelse: Sociologisk Institut, Linnésgade 22, $1361 \mathrm{~K}$.

CHRISTOPHER TILLEY (ed.): Interpretative Archaeology. Explorations in Anthropology Series. Oxford \& Providence, R.I.: Berg Publishers 1994. 210 sider. ISBN 0-85496842-3 hardback, pris $£ 54.95$. ISBN 0-85496850-4 paperback, pris $£ 19.95$.

JENS TONBOE: Rummets sociologi: Kritik af teoretiseringen af den materielle omverdens betydning i den sociologiske og den kulturgeografiske tradition. København: Akademisk Forlag 1993. 576 sider.

GARRY W. TROMPF: Payback: The Logic of Retribution in Melanesian Religions. Cambridge, New York \& Melbourne: Cambridge University Press 1994. 545 sider. ISBN 0-521-41691-4 hardback, pris £37.50.

JAMES W. TRULLINGER: Village at War: An Account of Conflict in Vietnam. Stanford: Stanford University Press 1994 [1980]. Distributed by Cambridge University Press. 235 sider, illustreret. ISBN 0-8047-2135-1 paperback, pris $£ 10.95$.
UDVIKLING, KULTUR, CIVILISATION. Den Jyske Historiker nr. 66. Århus: Aarhus Universitetsforlag 1994. 135 sider. Pris $125 \mathrm{kr}$.

VERDENSKULTUR - Verdenskulturtiårets Nyhedsbrev nr. 5, december 1993. Tema: Turisme. 24 sider, illustreret. Gratis. Henvendelse: Kulturtiårets Sekretariat, Vester voldgade 117, 1552 København V.

RICHARD J. WALTER: Politics and Urban Growth in Buenos Aires 1910-1942. Cambridge Latin American Studies 74. Cambridge, New York \& Melbourne: Cambridge University Press 1993. 278 sider, illustreret. ISBN 0-521-44165-X hardback, pris £35.00.

FIONA WILSON \& FINN STEPPUTAT (eds.): People in Politics - Debating Democracy in Latin America. Copenhagen: Centre for Development Research 1994. 127 sider. Pris DKK 80.

ANNE ELIZABETH YENTSCH: $A$ Chesapeake Family and Their Slaves: A Study in Historical Archaeology. Cambridge, New York \& Melbourne: Cambridge University Press 1994. 433 sider, illustreret. ISBN 0-521-43293-6 hardback $£ 55.00$. ISBN 0521-46730-6 paperback, £24.95. 
